\title{
OCORRÊNCIA DE ESPÉCIES NATIVAS E EXÓTICAS NOS QUINTAIS AGROFLORESTAIS URBANOS DE BREU BRANCO-PA
}

\author{
Alexandre Leão Gonçalves ${ }^{1}$; Vanessa Maria Silva da Cruz ${ }^{1}$; Alisson Rodrigo Souza \\ Reis²; Jamerson Rodrigo dos Prazeres Campos². \\ ${ }^{1}$ Discente de Engenharia Florestal, Universidade Federal do Pará \\ (eng.alexandregoncalves@outlook.com) \\ 2Professor Doutor, Faculdade de Engenharia Florestal-Universidade Federal do Pará \\ Recebido em: 08/04/2017 - Aprovado em: 10/06/2017 - Publicado em: 20/06/2017 \\ DOI: 10.18677/EnciBio_2017A79
}

\begin{abstract}
RESUMO
Os quintais agroflorestais apresentam uma constante dinamização ecológica entre espécies nativas e exóticas, de modo que essas relações podem explicar mudanças ocorridas ou que ocorrerão nos ecossistemas de uma região. O objetivo desse estudo foi avaliar a ocorrência de espécies nativas do território brasileiro e exóticas em quintais agroflorestais urbanos, no município de Breu Branco-PA. Entrevistaramse os proprietários dos quintais em dois bairros, utilizando um questionário com perguntas abertas e fechadas, indagando sobre o nome vernacular e a forma de uso das espécies, procedência da muda, e ainda observou-se a forma de manejo das plantas. As plantas foram identificadas em campo, com o auxílio do proprietário de cada quintal, sendo também fotografadas detalhadamente e comparadas com exsicatas disponíveis nas plataformas Flora do Brasil e MOBOT. Calculou-se a porcentagem dos tópicos do questionário, e os parâmetros fitossociológicos das espécies. O total de quintais pesquisados foi de 158, onde identificou-se 138 espécies, sendo 38 nativas e 100 exóticas. Do total, 96\% das plantas receberam o conceito Bom em relação ao manejo, $62 \%$ total de espécies são advindas de doação, e tanto as espécies nativas quanto as exóticas são utilizadas majoritariamente para a alimentação. Dessa maneira, constatou-se a predominância das espécies de origem exótica, em especial, as frutíferas de uso alimentar, seguindo o mesmo padrão encontrado nos quintais da região amazônica.
\end{abstract}

PALAVRAS-CHAVE: dinâmica ecológica, origem, usos.

\section{OCCURRENCE OF NATIVE AND EXOTIC SPECIES IN AGROFORESTRY BACKYARDS URBAN OF BREU BRANCO-PA}

\begin{abstract}
The agroforestry backyards have a constant dynamic between eco-native and exotic species, so that these relationships can explain the changes that have occurred or that will occur in ecosystems of the region. Thus, the objective of this study was to evaluate the occurrence of species native to the Brazilian territory and exotic in backyards urban agroforestry, in the municipality of Breu Branco-PA. Interviewed the owners of backyards in two neighborhoods, using a questionnaire with open and closed questions, inquiring about the name vernacular and the use of species, origin of the changes, and yet there was a plant health of the plants. The plants were identified in the field, with the help of the owner of each yard, being also
\end{abstract}


photographed in detail and compared with exsicatas available in the platforms Flora of Brazil and MOBOT. We calculated the percentage of topics in the questionnaire, and the phytosociological parameters of the species. The total number of backyards surveyed was 158, where 138 species were identified, being 38 native and 100 exotic. Of the total, $96 \%$ of plants received the Good concept of management, $62 \%$ of all species come from donation, and both native and exotic species are used mainly for food. Given that, there was a predominance of species of origin exotic, in particular, the seeds of food use, following the same pattern found in the backyards of the Amazon region.

KEYWORDS: ecological dynamics, origin, uses.

\section{INTRODUÇÃO}

Os Sistemas Agroflorestais (SAFs) consistem em formas de uso da terra que integram espécies perenes lenhosas com culturas agrícolas e/ou pecuária em arranjos espaciais e temporais (MAY \& TROVATTO, 2008). Na região amazônica, os SAFs têm sido recomendados como uma forma sustentável de uso da terra (SILVA et al., 2016), sendo considerados ecologicamente mais próximos dos sistemas agrícolas sustentáveis (PAULA et al., 2016), abrangendo diversas modalidades de cultivo. Dentre essas, destacam-se os quintais agroflorestais.

Os quintais agroflorestais (QAFs) são áreas ao redor da residência, no meio urbano, periurbano ou rural, onde ocorre a associação de espécies florestais, agrícolas, medicinais e animais, com o objetivo de fornecer complementação nutricional, remédios caseiros, renda e várias formas de bens e serviços (LUNZ, 2007; FREITAS et al., 2012).

Os QAFs desempenham importantes funções ecológicas, pois contribuem na conservação de plantas, asseguram a variabilidade genética, constituindo-se como importante banco de germoplasma (JUNIOR et al., 2013), e representam sistemas sustentáveis e mais resistentes a pragas (FERREIRA \& SABLAYROLLES, 2009).

Em razão das diferentes maneiras de cultivo e diversas formas de uso das plantas, ocorre uma dinâmica ecológica entre espécies nativas e exóticas nesses espaços (KABIR \& WEBB, 2008). Devido a essa dinamização, alguns estudos sugerem que esses agroecossistemas podem contribuir para a conservação de plantas nativas, bem como para a elaboração de estratégias que visem 0 uso sustentável dos recursos naturais (DUQUE-BRASIL et al., 2011).

Os quintais utilizam o método sucessional de espécies, preservando uma gama de plantas nativas, e assemelhando-se aos ambientes de floresta (SIVIERO et al., 2011). Apesar da relevância se avaliar os QAFs como espaço de conservação para as espécies nativas, há a necessidade de investigar a ocorrência de espécies exóticas, visto que isso pode explicar mudanças ocorridas na flora de uma dada região, e ainda pode contribuir com pesquisas posteriores (MORO et al., 2012).

Dessa maneira, os estudos em quintais agroflorestais são importantes para a compreensão do papel que os mesmos possuem na conservação de plantas, bem como para observar o modo de inserção de espécies exóticas e o seu manejo, tendo em vista os malefícios ou benefícios que isso pode ocasionar. Além disso, torna-se possível conhecer os produtos oriundos desses espaços e a sua relevância do ponto de vista de produção, seja em relação a espécies nativas ou exóticas.

Neste contexto, registra-se a importância de estudos com vistas à compreensão da dinâmica entre espécies vegetais nesses agroecossistemas, que apesar de suas diversas funcionalidades, têm recebido pouca atenção (AMARAL \& NETO, 2008), principalmente, àqueles situados nas zonas urbanas brasileiras 
(VIEIRA et al., 2012). Sendo assim, o presente estudo teve o objetivo de avaliar a ocorrência de espécies nativas do território brasileiro e exóticas em quintais agroflorestais urbanos, no município de Breu Branco - PA.

\section{Área de estudo}

\section{MATERIAL E MÉTODOS}

O estudo foi realizado na zona urbana do município de Breu Branco, situado na região sudeste do estado do Pará, sob as coordenadas geográficas: latitude 040ㄴ'04"S e longitude 493' $13^{\prime \prime O}$ (Figura 1). O município é pertencente à região de integração do lago de Tucuruí (FAPESPA, 2014), está a uma distância de $419 \mathrm{~km}$ da capital do estado, e possui uma população estimada em 62.737 mil habitantes, distribuída em $3.941,911 \mathrm{~km}^{2}$ de extensão territorial, com densidade demográfica de 13,32 hab/km² (IBGE, 2016).

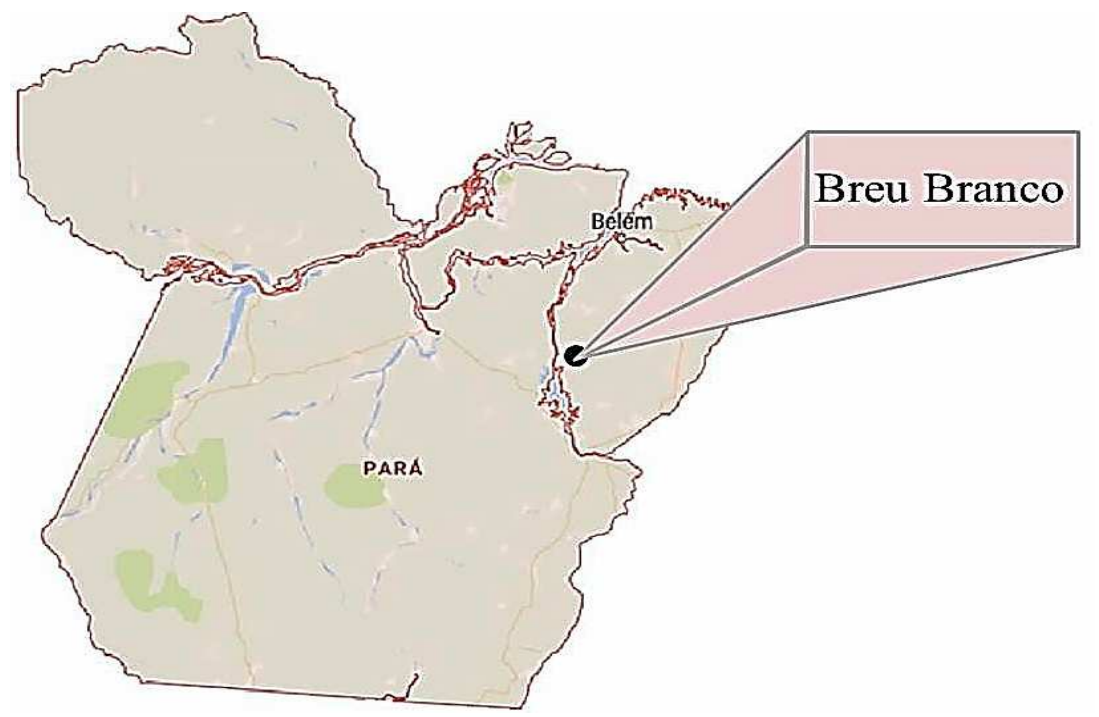

FIGURA 1. Localização geográfica do município de Breu Branco - PA.

Fonte: Adaptado de IBGE (2016).

A vegetação predominante no território breuense é do tipo Floresta Ombrófila Densa, com subtipo Ombrófila Aluvial, e ocorrência de Floresta Ombrófila mesclada com vegetação secundária, e as maiores unidades de mapeamento de solos no município correspondem aos Latossolos Amarelos (BREU BRANCO, 2006). O clima é tropical úmido, segundo a classificação de Köeppen, do subtipo Am, com índice pluviométrico anual da ordem de $2000 \mathrm{~mm}$, tendo moderado período de estiagem (entre julho e setembro), com precipitações inferiores a $50 \mathrm{~mm}$ e temperatura média de $26^{\circ} \mathrm{C}$ (PDRS, 2013).

\section{Coleta de dados}

Os dados foram coletados nos bairros: Novo Horizonte e Bela Vista, selecionando de forma aleatória dois quintais em cada quadra. Foram realizadas entrevistas com os proprietários dos quintais agroflorestais, tendo o auxilio de um questionário estruturado com perguntas abertas e fechadas, contendo indagações sobre o nome vernacular e a forma de uso das espécies, a procedência da muda (doação, compra, muda produzida pelo cultivador) e o manejo das plantas. Todos os dados foram levantados com o consentimento prévio dos proprietários dos QAFs investigados. 
As plantas citadas foram enquadradas nas seguintes categorias de uso: alimentar, medicinal, venda, produção de sombra e uso religioso. O manejo de cada planta foi classificado nos conceitos: Bom (recebe adubação, irrigação e poda), Regular (recebe apenas um ou dois tipos de trato) e Ruim (não recebe trato).

Para a observação dos aspectos estruturais, realizou-se a medição do terreno dos quintais, bem como a medição e a quantificação de todos os indivíduos com diâmetro a $1,30 \mathrm{~cm}$ do solo maior ou igual a cinco centímetros (DAP $\geq 5 \mathrm{~cm}$ ). $\mathrm{E}$ ainda, classificou-se as espécies inventariadas quanto ao seu hábito de crescimento.

Com o auxílio do proprietário de cada quintal, as plantas encontradas no local foram identificadas em campo. As plantas também foram fotografadas detalhadamente e comparadas com exsicatas disponíveis nas bases de dados das plataformas Flora do Brasil (FORZZA et al., 2016) e Missouri Botanical Garden (MOBOT, 2016). Outras ferramentas utilizadas foram chaves de identificação (SOUZA \& LORENZI, 2012; LORENZI, 2014), e levantamento bibliográfico (GONÇALVES \& LORENZI, 2011; MARTINS, 2012; MACHIORI, 2013; BOARI et al., 2014).

As buscas realizadas junto às plataformas Flora do Brasil e MOBOT também foram úteis na obtenção de informações sobre a origem e distribuição geográfica de determinadas espécies. O sistema taxonômico adotado foi o APG III (2009).

\section{Análise dos dados}

A partir dos dados levantados nos QAFs, os indivíduos que atenderam o critério de inclusão de DAP foram agrupados em classes diamétricas. Essas foram definidas pelo método de distribuição de frequência para intervalos de classes, de acordo com VIEIRA (2012).

Cada quintal foi considerado como uma unidade amostral, assim, calculou-se a porcentagem dos tópicos abordados no questionário, e os parâmetros fitossociológicos das espécies inventariadas: frequência relativa $(F R)$, densidade relativa (DR), dominância relativa (DoR), Índice de Valor de Importância (IVI) e o Índice de Valor de Cobertura (IVC) conforme os métodos de MUELLER-DOMBOIS \& ELLENBERG (1974). Os cálculos e o processamento dos dados foram feitos através do software Excel 2013.

\section{RESULTADOS E DISCUSSÃO}

O total de quintais agroflorestais pesquisados foi de 158, sendo 116 no bairro Novo Horizonte, e 42 no Bela Vista em razão do mesmo possuir menor número quadras em relação ao outro bairro estudado. O estudo registrou 2.208 indivíduos distribuídos em 56 famílias e 138 espécies.

DUQUE-BRASIL et al. (2011) estudando quintais agroflorestais em comunidades rurais do Parque Estadual da Mata Seca, obtiveram resultados inferiores ao desta pesquisa, identificando 1.267 indivíduos, 39 famílias botânicas e 87 espécies. Resultados inferiores também ocorreram nos trabalhos de VIEIRA et al. (2012) e JUNIOR et al. (2013).

A família botânica que mais se destacou foi a Lamiaceae (10 spp.), seguida por: Euphobiaceae (8 spp.), Fabaceae (8 spp.), Solanaceae (7 spp.), Arecaceae (6 spp.), Rutaceae (6 spp.), Cucurbitaceae ( 6 spp.) e Malvaceae (5 spp.). ALMEIDA \& GAMA (2014) também verificaram a família Lamiaceae entre as que continham o maior número de espécies, além dessa, as famílias Solanaceae, Rutaceae e Malvaceae. 
A predominância da família Lamiaceae é explicada pelas múltiplas utilidades que as espécies possuem para os cultivadores (alimentar, medicinal, religioso e outros), além disso, são plantas que podem ser cultivadas em pequenos espaços devido ao seu hábito de crescimento. De acordo com SOUZA \& LORENZI (2012), a família Lamiaceae é representada principalmente por espécies herbáceas, comumente as aromáticas, e no Brasil ocorrem cerca 38 gêneros e 500 espécies pertencentes a essa família.

Os quintais apresentaram área média de $343 \mathrm{~m}^{2}$, e das plantas encontradas nesses espaços $45 \%$ foram herbáceas, 23\% arbustivas, 22\% arbóreas, $6 \%$ arvoretas, $2 \%$ trepadeiras, e as subarbustivas e lianas somaram $1 \%$ cada uma. SIVIEIRO et al. (2011) registraram uma área média de 397,9 $\mathrm{m}^{2}$ nos QAFs urbanos de Rio Branco, Acre. Discrepante aos resultados desse estudo, esses autores verificaram as arbóreas como o hábito de crescimento predominante (38\%), já as herbáceas somaram $27,8 \%$ do total de espécies inventariadas. Em relação à distribuição diamétrica, observou-se que a maioria dos indivíduos se enquadraram nas menores classes diamétricas (figura 2).

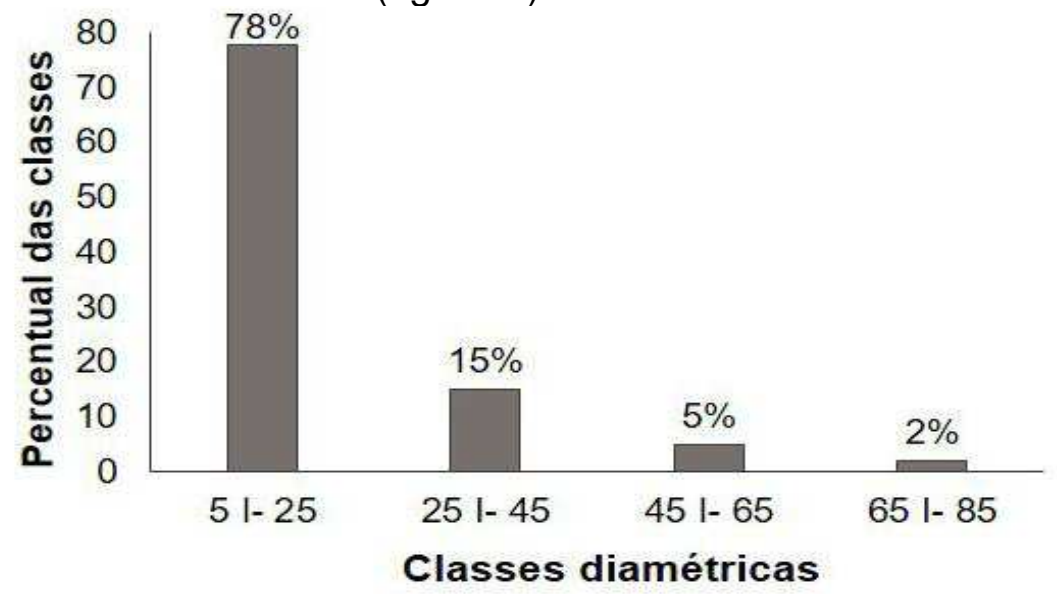

FIGURA 2. Distribuição diamétrica dos indivíduos inventariados nos QAFs urbanos de Breu Branco - PA.

Assim como na análise do hábito de crescimento (48\% herbáceas), esse resultado está associado, principalmente, à localização desses quintais, haja vista que fatores como o tamanho do quintal em área urbana e o incômodo que as plantas podem causar aos vizinhos (danos ao patrimônio com a queda de galhos e/ou frutos no telhado, queda de folhas no quintal vizinho, sombreamento em local indesejado), tendem a limitar o cultivo de espécies de grande e médio porte.

De modo semelhante a este estudo, GONÇALVES et al. (2015) investigando quintais agroflorestais de uma comunidade rural em Santa Bárbara do Pará, também registraram a maioria dos indivíduos nas menores classes diamétricas, obtendo $66 \%$ na classe 3-10 cm, 33\% na classe 10,1-18 cm, e 1\% na classe 18,1-26 cm. Apesar dessa similaridade, deve-se considerar que os indivíduos registrados nos QAFs urbanos de Breu Branco alcançaram diâmetros superiores (individuo de maior DAP= $85 \mathrm{~cm}$ ) aos de Santa Bárbara do Pará (individuo de maior DAP $=26 \mathrm{~cm}$ ).

No estado do Acre, LIMA \& LEÃO (2013) obtiveram DAP mínimo de $5,7 \mathrm{~cm}$ e máximo de $60,9 \mathrm{~cm}$ num inventário realizado em área de floresta plantada com espécies nativas. Em uma floresta de várzea no município de Ananindeua, estado do Pará, ALMEIDA \& JARDIM (2011) verificaram a maior concentração de indivíduos (860 no total) numa classe que variou de $17,7 \mathrm{~cm}$ a $25,2 \mathrm{~cm}$ de DAP. 
Em geral, nota-se que as classes diamétricas podem variar em escalas que vão de acordo com o ambiente onde os indivíduos estão inseridos, e que mesmo assim, podem apresentar semelhança com classes diamétricas de indivíduos de ambientes diferentes.

Em se tratando das formas de uso das espécies, predominou o alimentar $(81 \%)$, seguido pelo uso medicinal $(15 \%)$, produção de sombra $(2 \%)$, sendo que as categorias venda e uso religioso tiveram o mesmo percentual ( $1 \%$ cada categoria). $\mathrm{Na}$ figura 3 é possível observar a ocorrência de espécies nativas e exóticas nas diferentes categorias de uso.

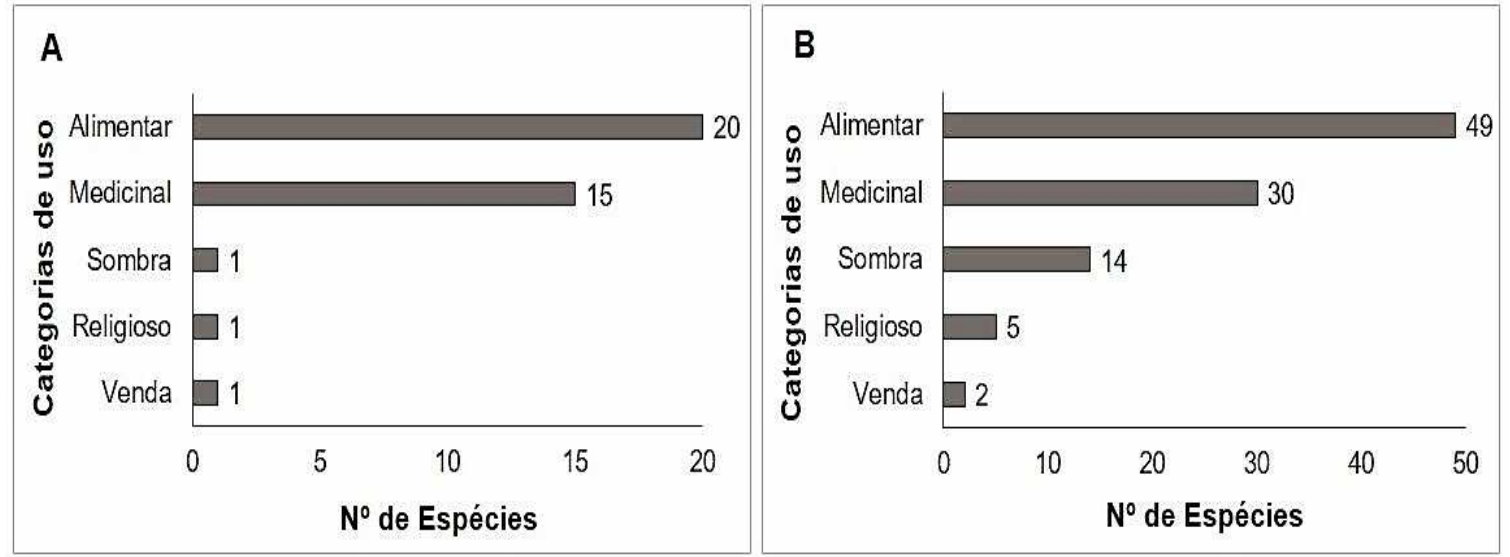

FIGURA 3. Número de espécies nativas (A) e exóticas (B) identificadas nos QAFs urbanos de Breu Branco - PA, de acordo com a categoria de uso.

Dentre as espécies utilizadas na alimentação, destacaram-se: o açaí (Euterpe oleracea Mart), o quiabo (Abelmoschus esculentus (L.) Moench), a manga (Mangifera indica L.) e a acerola (Malphigia glabra L.). Essas espécies se mostraram importantes na alimentação dos proprietários de quintais, além disso, verifica-se uma preferência gastronômica pelos frutos gerados por essas plantas.

O mesmo resultado sobre a predominância da categoria de uso alimentar foi obtido por DUQUE-BRASIL et al. (2011), VIEIRA et al. (2012) e ALMEIDA \& GAMA (2014), ambos em quintais da zona rural, e por AMARAL \& NETO (2008) e SIVIERO et al. (2011) em quintais urbanos.

Através desses registros, somados aos obtidos nos QAFs urbanos de Breu Branco, é possível inferir sobre a importância dos quintais agroflorestais como espaço de produção de alimento, tendo grande relevância na segurança alimentar das famílias, seja em área rural ou urbana. Do universo de plantas inventariadas, constatou-se que o número de espécies exóticas foi superior ao de nativas do Brasil (figura 4).

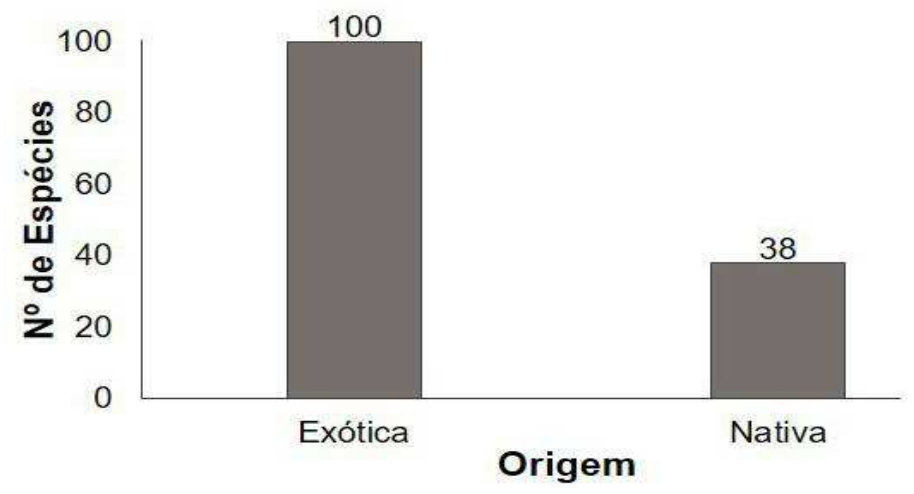

FIGURA 4. Quantificação das espécies identificadas nos QAFs urbanos de Breu Branco - PA, segundo o seu grupo de origem. 
Observa-se que é corriqueiro que os resultados de pesquisas em quintais agroflorestais apontem as espécies exóticas como as mais frequentes. Assim como ocorreu nos QAFS urbanos de Breu Branco, FLORENTINO et al. (2007) encontraram maior percentual de espécies exóticas nos quintais agroflorestais de Caruaru, estado de Pernambuco.

Na pesquisa realizada por SIVIERO et al. (2011), 82,3\% do total de plantas identificadas eram de origem exótica. LUNZ (2007) investigando o cultivo de frutíferas nos quintais agroflorestais de assentamentos rurais em Rondônia, também constatou a maior abundância das exóticas, dentre as 50 espécies inventariadas, 32 foram exóticas e apenas 18 nativas.

Essa sobreposição das espécies exóticas nos quintais de Breu Branco está associada, principalmente, ao fato das espécies serem majoritariamente representadas pelas frutíferas de origem exótica, que fazem parte do hábito alimentar das famílias dos cultivadores, de modo que possuem um papel importante na subsistência das mesmas. ALMEIDA \& GAMA (2014) sugerem que aspectos da miscigenação de culturas, que por sua vez é justificada pela imigração, exercem influência na inserção de plantas exóticas em diversas regiões.

Para DUQUE-BRASIL et al. (2011), os quintais são locais importantes para incremento da diversidade vegetal ao admitirem a livre incorporação de espécies exóticas para atender demandas do mantenedor. Esses autores inferem que esses espaços também podem contribuir para a conservação da biodiversidade, exercendo a função de refúgio de espécies nativas, endêmicas e/ou ameaçadas de extinção. Já MORO et al. (2012) chamam a atenção para o risco que há em introduzir espécies exóticas nos ecossistemas, em razão da sua adaptabilidade e dispersão, que podem torna-las invasoras e causar danos severos a biodiversidade local.

A análise do manejo evidenciou que $96 \%$ das plantas receberam o conceito Bom (dispõem de adubação, irrigação e poda), as que receberam conceito Regular ou Ruim ambas somaram $2 \%$. Quanto à procedência da muda, constatou-se que a maioria é advinda de doação (62\%), outra parte foi produzida pelo cultivador $(28 \%)$ e uma pequena parcela foi comprada (10\%). Corroborando, FLORENTINO et al. (2007) obtiveram resultados semelhantes em relação a procedência das plantas, sendo que maioria dos casos, as plantas foram trazidas, trocadas ou doadas por vizinhos, amigos ou parentes.

O fato da maioria dessas plantas serem de doação, somado ao bom tratamento que recebem, possui influência na dispersão/inserção de diversas espécies no âmbito dos quintais, uma vez que a interação entre vizinhos, amigos e parentes possibilita a doação ou troca de plantas por interesses de uso, e ao mesmo tempo essas interações ocorrem sem qualquer noção do risco que a inserção de espécies exóticas pode oferecer ao ecossistema local. Vale ressaltar que isso também pode favorecer a conservação de espécies nativas, e todos esses fatores necessitam de maiores investigações para serem melhor compreendidos.

Nos quintais investigados registrou-se uma quantidade significativa de espécies, porém a maioria delas apresentou baixa frequência. Dessa maneira, as espécies nativas do Brasil e exóticas que mais se destacaram em frequência, sua classificação botânica e formas de uso, com seus respectivos parâmetros fitossociológicos estão representadas nas tabelas 1 e 2, respectivamente. 
TABELA 1. Espécies nativas do Brasil c/ maior ocorrência nos QAFs urbanos de Breu Branco e seus parâmetros fitossociológicos.

\begin{tabular}{ccccccccccc}
\hline FAMíLIA & ESPÉCIE & VERNÁCULO & USO & No & FR & DR & DoR & IVI & IVC \\
\hline Arecaceae & Euterpe oleracea Mart. & Açaí & A & 162 & 36,9863 & 49,3902 & 87,6290 & 58,0018 & 68,5096 \\
Euphobiaceae & Manihot esculenta Crantz & Macaxeira & A & 55 & 8,2192 & 16,7683 & - & 8,3292 & 8,3841 \\
Malvaceae & Theobroma grandiflorum (Willd. ex. & Cupuaçu & A & 42 & 19,1781 & 12,8049 & 3,3318 & 11,7716 & 8,0683 \\
Apiaceae & Spreng) K.Schum. & Eryngium foetidum L. & Chicória & A & 24 & 16,4384 & 7,3171 & - & 7,9185 & 3,6585 \\
Arecaceae & Bactris gasipaes Kunth & Pupunha & A & 23 & 10,9589 & 7,0122 & 8,9881 & 8,9864 & 8,0002 \\
Myrtaceae & Plinia cauliflora (Mart.) Kausel & Jabuticaba & A & 22 & 8,2192 & 6,7073 & 0,0511 & 4,9925 & 3,3792 \\
\hline TOTAL & & & & $\mathbf{3 2 8}$ & $\mathbf{1 0 0}$ & $\mathbf{1 0 0}$ & $\mathbf{1 0 0}$ & $\mathbf{1 0 0}$ & $\mathbf{1 0 0}$ \\
\hline A & & & &
\end{tabular}

A = Alimentar; № = Número de indivíduos da espécie; FR = Frequência Relativa (\%); DR = Densidade Relativa (\%); DoR = Dominância Relativa (\%); IVI = Índice de Valor de Importância (\%); IVC = Índice de Valor de Cobertura (\%).

TABELA 2. Espécies exóticas c/ maior ocorrência nos QAFs urbanos de Breu Branco e seus parâmetros fitossociológicos.

\begin{tabular}{|c|c|c|c|c|c|c|c|c|c|}
\hline FAMÍLIA & ESPÉCIE & VERNÁCULO & Uso & № & $\mathbf{F R}$ & DR & DoR & IVI & IVC \\
\hline Malvaceae & $\begin{array}{l}\text { Abelmoschus esculentus (L.) } \\
\text { Moench }\end{array}$ & Quiabo & A & 128 & 10,4603 & 28,5078 & - & 12,9893 & 14,2539 \\
\hline Anacardiaceae & Mangifera indica L. & Manga & A & 98 & 23,8494 & 21,8262 & 95,3821 & 47,0192 & 58,6042 \\
\hline Malphigiaceae & Malphigia glabra L. & Acerola & A & 70 & 19,6653 & 15,5902 & 2,3091 & 12,5215 & 8,9496 \\
\hline Rutaceae & Citrus limon (L.) Osbeck & Limão & A & 68 & 18,4100 & 15,1447 & 0,9393 & 11,4979 & 8,0418 \\
\hline Liliaceae & Allium schoenoprasum L. & Cebolinha & A & 46 & 17,9916 & 10,2452 & - & 9,4122 & 5,1224 \\
\hline Annonaceae & Annona squamosa L. & Ata & A & 39 & 9,6234 & 8,6859 & 1,3696 & 6,5599 & 5,0281 \\
\hline TOTAL & & & & 449 & 100 & 100 & 100 & 100 & 100 \\
\hline
\end{tabular}

$\mathrm{A}=$ Alimentar; № = Número de indivíduos da espécie; FR = Frequência Relativa (\%); DR = Densidade Relativa (\%); DoR = Dominância Relativa (\%); IVI = Índice de Valor de Importância (\%); IVC = Índice de Valor de Cobertura (\%). 
Dentre as nativas do Brasil, Euterpe oleracea foi a espécie que apresentou maior IVI e IVC, alcançando altos valores em todos os parâmetros fitossociológicos avaliados. Segundo LAMPRECHT (1990), os elevados valores de frequência, densidade, e dominância representam as espécies mais importantes, quando o levantamento em questão é a estrutura da comunidade, com vistas à observação da fisionomia desse ambiente.

Em uma pesquisa sobre os Sistemas Agroflorestais do município de Santa Maria do Pará, RAIOL \& ROSA (2012) verificaram que Euterpe oleracea está entre as espécies de maior interesse de cultivo para os agricultores locais. Essa espécie também alcançou alto índice de valor de importância no trabalho de ALMEIDA \& GAMA (2014), e maior frequência na pesquisa de GONÇALVES et al. (2015). Já SIVIERO et al. (2014) registraram o uso ornamental de E. oleracea nos quintais urbanos de Rio Branco, Acre.

Neste sentido, VIEIRA et al. (2012) enfatizam que o açaí (Euterpe oleracea) é uma espécie de uso alimentar muito apreciada na Amazônia. Os autores expõem que o processamento do fruto de E. oleracea produz uma bebida chamada de "vinho do açaí", que possui valor nutritivo e terapêutico, e faz parte do hábito alimentar na região amazônica, assim como a macaxeira (Manihot esculenta).

No conjunto das espécies exóticas Abelmoschus esculentus e Mangifera indica foram as mais frequentes. De maneira oposta a esses resultados, SANTOS et al. (2013) identificaram pouca ocorrência de $A$. esculentus nos quintais de Itaporanga d'Ajuda-SE, mesmo estes cultivos tendo fins comercias e de subsistência para os agricultores desse município. A baixa frequência dessa espécie também foi verificada por RAIOL \& ROSA (2012).

Corroborando com os resultados dessa pesquisa, JUNIOR et al. (2013) registraram a $M$. indica entre as espécies de maior ocorrência nos QAFs do PDS Virola Jatobá, em Anapú-PA. Outros trabalhos como os de LUNZ (2007) e FLORENTINO et al. (2007) obtiveram o mesmo resultado em relação a alta frequência dessa espécie.

Este resultado está associado principalmente ao uso alimentar do fruto de $M$. indica. Essa espécie produz um fruto suculento que é bastante consumido em grande parte do Brasil, além disso, a sua copa fornece sombra abundante, e em muitas cidades é utilizada para fins estéticos na arborização urbana.

De modo geral, os quintais mostraram-se importantes do ponto de vista da produção, tanto em relação às espécies nativas quanto às exóticas. As espécies de ambas as origens fornecem produtos que fazem parte da culinária, dos cuidados com a saúde e da cultura e bem-estar dos cultivadores dessas plantas, e ainda, geram a possibilidade de se tornarem uma fonte de renda.

Já do ponto de vista da conservação, a relevância está na proteção que esses agroecossistemas podem proporcionar para as espécies nativas e/ou endêmicas, bem como para aquelas que estejam sob ameaça de extinção, seja nativa ou exótica. Isso se torna possível devido à liberdade de inserção de espécies nos quintais agroflorestais, em função do interesse de uso pelos mantenedores. Assim, observa-se que os QAFs possuem grande importância tanto no âmbito da produção como no que se refere a conservação.

\section{CONCLUSÃO}

Os quintais agroflorestais urbanos de Breu Branco possuem semelhança com o padrão encontrado nos quintais da região amazônica, cujo ocorre a predominância 
de espécies de origem exótica, em especial, as frutíferas de uso alimentar, que são essenciais para a alimentação e subsistência das famílias dos cultivadores.

Apesar da maior expressividade das exóticas, cultiva-se uma gama de espécies nativas do território brasileiro, dando indicativos que esses espaços podem contribuir de maneira significativa para a conservação de plantas da flora brasileira, servindo como refúgio e auxiliando na manutenção de espécies que possuem um papel fundamental no ecossistema local.

\section{REFERÊNCIAS}

ALMEIDA, A. F.; JARDIM, M. A. G. Florística e estrutura da comunidade arbórea de uma floresta de várzea na llha de Sororoca, Ananindeua, Pará, Brasil. Scientia Forestalis, Piracicaba, v. 39, n. 90, p.191-198, jan./mai. 2011. Disponível em: <http://www.ipef.br/publicacoes/scientia/nr90/cap07.pdf>. Acesso em: 11 nov. 2016.

ALMEIDA, L. S. de; GAMA, J. R. V.; Quintais agroflorestais: Estrutura, composição florística e aspectos socioambientais em área de assentamento rural na Amazônia brasileira. Revista Ciência Florestal, Santa Maria, v. 24, n. 4, p. 1041-1053, out./dez. 2014. Disponível em: <http://dx.doi.org/10.5902/1980509816617>. doi: 10. 5902/1980509816617.

AMARAL, C. N. do; NETO, G. G. Os quintais como espaço de conservação e cultivo de alimentos: um estudo na cidade de Rosário Oeste (Mato Grosso, Brasil). Boletim Museu Paraense Emílio Goeldi, Belém, v. 3, n. 3, p. 329-341, set./dez. 2008. Disponível em: <http://dx.doi.org/10.1590/S1981-81222008000300004>. doi: 10.159 0/S1981-81222008000300004.

ANGIOSPERM PHYLOGENY GROUP. An update of the angiosperm phylogeny group classification for the orders and families of flowering plants: APG III. Botanical Journal of the Linnean Society, v. 141, n. 4, p. 399-436, 2003. Disponível em: <http://dx.doi.org/10.1046/j.1095-8339.2003.t01-1-00158.x>. doi: 10.1046/j.1095-833 9.2003.t01-1-00158.x.

BOARI, A. de J.; SANTOS, A. F. dos; BARBOSA, A. P.; VIEIRA, B. de A. H.; TREMACOLDI, C. R.; AUER, C. G.; LUSTOSA, D. C. REZENDE, D. V de; CARVALHO, E. de A. Doenças de espécies florestais arbóreas nativas e exóticas na Amazônia. v. 1. 1 ed. Brasília: EMBRAPA, 2014. 209 p.

BREU BRANCO. Plano diretor do município de Breu Branco. Prefeitura Municipal de Breu Branco, 2006. Disponível em: <http://www.seidurb.pa.gov.br/pdm/breu_bran co/Plano_Diretor.pdf>. Acesso em: 18 out. 2016.

DUQUE-BRASIL, R. D.; SOLDATI, G. T.; SANTOS, M. M. E.; REZENDE, M. Q.; NETO, S. D. A.; COELHO, F. M. G. Composição, uso e conservação de espécies arbóreas em quintais de agricultores familiares na região da mata seca nortemineira, Brasil. Revista Sitientibus série Ciências Biológicas, Feira de Santana, v. 11, n. 2, p. 287-297, jun./ago. 2011. Disponível em: <http://dx.doi.org/10.13102/scb7 6>. doi: $10.13102 / \mathrm{scb} 76$. 
FAPESPA. Diagnóstico Socioeconômico e Ambiental da Região de Integração Lago de Tucuruí. Fundação Amazônica de Amparo a Estudos e Pesquisas do Pará, 2014. Disponível em: <http://www.fapespa.pa.gov.br/sites/default/files/Regiao_Integr acao_Lago_de_Tucurui.pdf>. Acesso em: 15 dez. 2016.

FERREIRA, T. B.; SABLAYROLLES, M. das G. P. Quintais agroflorestais como fonte de saúde: plantas medicinais na Comunidade de Vila Franca, Reserva Extrativista Tapajós-Arapiuns, Pará. Revista Brasileira de Agroecologia, São Paulo, v. 4, n. 2, p. 59-68, nov. 2009. Disponível em: <http://www.aba-agroecologia.org.br/revistas/ind ex.php/rbagroecologia/article/view/8955/6269>. Acesso em: 9 nov. 2016.

FLORENTINO, A. T. N.; ARAÚJO, E. de L.; ALBUQUERQUE, U. P. de. Contribuição de quintais agroflorestais na conservação de plantas da Caatinga, Município de Caruaru, PE, Brasil. Revista Acta Botânica Brasilica, Belo Horizonte, v. 21, n. 1, p. 37-47, jan./mar. 2007. Disponível em: <http://dx.doi.org/10.1590/S0102-3306200700 0100005>. doi: 10.1590/S0102-33062007000100005.

FORZZA, R. C.; COSTA, A. F da; WALTER, B. M. T.; BICUDO, C.; MOURA, C. W. N.; ZAPPI, D.; PERALTA, D. F. Lista de espécies da flora do Brasil. 2016. Disponível em: <http://floradobrasil.jbrj.gov.br>. Acesso em: 12 nov. 2016.

FREITAS, A. V. L.; COELHO, M. F. B.; MAIA, S. S. S.; AZEVEDO, R. A. B. Plantas medicinais: um estudo etnobotânico nos quintais do Sítio Cruz, São Miguel, Rio Grande do Norte, Brasil. Revista Brasileira de Biociências, Porto Alegre, v. 10, n. 1, p. 48-59, jan./mar. 2012. Disponível em: <http://www.ufrgs.br/seerbio/ojs/index.ph p/rbb/article/view/1833/1093 >. Acesso em: 10 nov. 2016.

GONÇALVES, E. G.; LORENZI, H. Morfologia Vegetal: organografia e dicionário ilustrado de morfologia das plantas vasculares. v. 1. 2 ed. São Paulo: Instituto Plantarium de estudos da flora, 2011. 544 p.

GONÇALVES, J. P.; SOUZA, L. P. F. de; SOUZA, N. H. de; MIRANDA, T. G.; PAULA, M. T. de. Análise florística e estrutural de quintais agroflorestais na comunidade expedito ribeiro em Santa Bárbara do Pará. Revista Enciclopédia Biosfera, Goiânia, v. 11, n. 22, p. 173-183, nov./dez. 2015. Disponível em: <http://dx.doi.org/10.18677/Enciclopedia_Biosfera_2015_079>.doi: 10.18677/Encicl opedia_Biosfera_2015_079.

IBGE. Estatísticas das cidades: Breu Branco, Pará. Instituto Brasileiro de Geografia Estatística, 2016. Disponível em: <http://cidades.ibge.gov.br/xtras/perfil.ph p?codmun=150178>. Acesso em: 17 dez. 2016.

JUNIOR, O. F.; HAMADA, M. O. de S.; SOUZA, O. de P. S. de; CORREA, R. F. Levantamento florístico dos quintais agroflorestais do PDS Virola Jatobá em Anapú, Pará. Revista Enciclopédia Biosfera, Goiânia, v. 9, n. 17, p. 1793-1805, jun. 2013. Disponível em: <http://www.conhecer.org.br/enciclop/2013b/CIENCIAS\%20AGRARI AS/Levantamento.pdf>. Acesso em: 12 nov. 2016. 
KABIR, E.; WEBB, E. L. Can homegardens conserve biodiversity in Bangladesh? Revista Biotropica, Belo Horizonte, v. 40, n. 1. P. 95-103. Disponível em: <http://dx.doi.org/10.1111/j.1744-7429.2007.00346.x>. doi: 10.1111/j.1744-7429.200 7.00346.x.

LIMA, J. P. da C.; LEÃO, J. R. A. Dinâmica de crescimento e distribuição diamétrica de fragmentos de florestas nativa e plantada na Amazônia sul oriental. Revista Floresta e Ambiente, Rio de Janeiro, v. 20, n. 1, p. 70-79, jan./mar. 2013. Disponível em: <http://dx.doi.org/10.4322/floram.2012.065>. doi: 10.4322/floram.201 2.065 .

LAMPRECHT, H.; Silvicultura nos trópicos: Ecossistemas florestais e respectivas espécies arbóreas - possibilidades e métodos de aproveitamento sustentado. Instituto de Silvicultura da Universidade Gottingen. Eschborn. Rep. Federal da Alemanha. 1990. 343p.

LORENZI, H. Árvores Brasileiras: manual de identificação e cultivo de plantas arbóreas nativas do Brasil. v. 1.6 ed. São Paulo: Instituto Plantarium de estudos da flora, 2014. 384 p.

LUNZ, A. M. P. Quintais agroflorestais e o cultivo de espécies frutíferas na Amazônia. Revista Brasileira de Agroecologia, Porto Alegre, v. 2, n. 2, p. 12551258, out. 2007. Disponível em: <http://www.aba-agroecologia.org.br/revistas/index.p hp/rbagroecologia/article/view/7290>. Acesso em: 13 nov. 2016.

MARCHIORI, J. N. C. Elementos de Dendrologia. v. 1. 3 ed. Santa Maria: UFSM, 2013. $216 \mathrm{p}$.

MARTINS, S. V. Ecologia de florestas tropicais do Brasil. v. 1. 2 ed. Viçosa: UFV, 2012. $371 \mathrm{p}$.

MAY, P. H.; TROVATTO, C. M. M. Manual agroflorestal para a Mata Atlântica. v. 1. 1 ed. Brasília: Ministério do Desenvolvimento Agrário, 2008. 196 p.

MOBOT. Missouri Botanical Garden. 2016. Disponível em: <http://www.tropicos.or g>. Acesso em: 15 jul. 2016.

MORO, M. F.; SOUZA, V. C.; FILHO, A. T. de O.; QUEIROZ, L. P. de; FRAGA, C. N. de; RODAL, M. J. N.; ARAÚJO, F. S. de; MARTINS, F. R. Alienígenas na sala: o que fazer com espécies exóticas em trabalhos de taxonomia, florística e fitossociologia? Revista Acta Botânica Brasílica, Belo Horizonte, v. 26, n. 4, p. 991-999, out./dez. 2012. Disponível em: <http://dx.doi.org/10.1590/S0102-33062012000400029>. doi: 10.1590/S0102-33062012000400029.

MUELLER-DOMBOIS, D.; ELLENBERG, H. Aims and methods of vegetation ecology. v. 1. 1 ed. New York: Blackburn Press, 1974. 547 p.

PAULA, P. D. de; CAMPELLO, E. F. C.; GUERRA, J. G. M.; SANTOS, G. de A.; RESENDE, A. S. de. Decomposição das podas de leguminosas arbóreas Gliricidia sepium e Acacia angustissima em um sistema agroflorestal. Revista Ciência 
Florestal, Santa Maria, v. 25, n. 3, p. 791-800, jul./set. 2015. Disponível em: <http://dx.doi.org/10.5902/1980509819696>. doi: 10.5902/1980509819696.

PDRS. Plano de desenvolvimento regional sustentável do lago de Tucuruí. Governo do estado do Pará, 2013. Disponível em: <http://www.mi.gov.br/c/document library/get_file?uuid=0a6eac82-0b58-40bf-a60e-c80828c0ec90\&groupld=10157>. Acesso em: 18 out. 2016.

RAIOL, C. S.; ROSA, L. dos S. Sistemas agroflorestais na Amazônia oriental: o caso dos agricultores familiares de Santa Maria do Pará, Brasil. Revista Brasileira de Ciências Agrárias, Recife, v. 8, n. 2, p. 258-265, dez. 2012. Disponível em: <http://dx.doi.org/ 10.5039/agraria.v8i2a2045>. doi: 10.5039/agraria.v8i2a2045.

SILVA, C. M. da; VASCONCELOS, S. S.; JUNIOR, M. M.; BISPO, C. J. C.; KATO, O. R.; JUNIOR, A. C. da S.; CASTELLANI, D. C. Variação temporal do efluxo de Co2 do solo em sistemas agroflorestais com palma de óleo na Amazônia Oriental. Revista Acta Amazônica, Manaus, v. 46, n. 1, p. 1-12, jan./mar. 2016. Disponível em: <http://dx.doi.org/10.1590/1809-4392201500193>. doi: 10.1590/1809-439220150019 3.

SIVIERO, A.; DELUNARDO, T. A.; HAVERROTH, M.; OLIVEIRA, L. C. de; MENDONÇA, A. M. S. Cultivo de espécies alimentares em quintais urbanos de Rio Branco, Acre, Brasil. Revista Acta Botânica Brasilica, Belo Horizonte, v. 25, n. 3, p. 549-556, mar./mai. 2011. Disponível em: <http://dx.doi.org/10.1590/S0102-33062011 000300006 >. doi: 10.1590/S0102-33062011000300006.

SIVIERO, A.; DELUNARDO, T. A.; HAVERROTH, M.; OLIVEIRA, L. C. de; ROMAN, A. L. C.; MENDONÇA, A. M. da S. Plantas ornamentais em quintais urbanos de Rio Branco, Brasil. Boletim do Museu Paraense Emílio Goeldi, Belém, v. 9, n. 3, p. 797-813, set./dez. 2014. Disponível em: <http://dx.doi.org/10.1590/1981-8122201400 0300015>. doi: 10.1590/1981-81222014000300015.

SOUZA, V. C.; LORENZI, H. Botânica Sistemática: guia ilustrado para identificação das famílias de fanerógamas nativas e exóticas no Brasil, baseado em APG III. v. 1. 3 ed. Nova Odessa: Instituto Plantarium de estudos da flora, 2012. 768 p.

VIEIRA, S. Estatística básica. v. 1. 1 ed. São Paulo: Cengage Learning, 2012.176p.

VIEIRA, T. A.; ROSA, L. dos S.; SANTOS, M. M. de L. S. Agrobiodiversidade de quintais agroflorestais no município de Bonito, estado do Pará. Revista Ciência Agrárias, Belém, v. 55, n. 3, p. 159-166, jul./set. 2012. Disponível em: <http://dx.doi. org/10.4322/rca.2012.054>. doi: 10.4322/rca.2012.054. 
TABELA COM TODAS AS ESPÉCIES INVENTARIDAS

\begin{tabular}{|c|c|}
\hline NOME CIENTÍFICO & NOME POPULAR \\
\hline Anacardium occidentale L. & Caju \\
\hline Ananas comosus (L.) Merril & Abacaxi \\
\hline Bactris gasipaes Kunth & Pupunha \\
\hline Bixa orellana $\mathrm{L}$. & Urucum \\
\hline Byrsonima crassifolia (L.) Kunth & Muruci \\
\hline Caesalpinia ferrea Mart. & Jucá \\
\hline Capsicum annuum L. & Pimentão \\
\hline Cariniana estrellensis (Raddi) Kuntze & Jequitibá \\
\hline Cenostigma macrophyllum Tul. & Maxarimbé \\
\hline Cereus jamacaru DC. & Mandacarú \\
\hline Cissus verticillata (L.) Nicolson \& C.E.Jarvis & Insulina \\
\hline Costus sp. & Canarana \\
\hline Costus spiralis (Jacq.) Roscoe & Cana manca \\
\hline Cucumis anguria $\mathrm{L}$. & Maxixe \\
\hline Duranta erecta L. & Pingo de ouro \\
\hline Eryngium foetidum L. & Chicória \\
\hline Euterpe oleracea Mart. & Açai \\
\hline Hancornia speciosa Gomes & Mangaba \\
\hline Jatropha curcas L. & Pinhão manso \\
\hline Jatropha gossypiifolia L. & Pião roxo \\
\hline Manihot esculenta Crantz & Macaxeira \\
\hline Manihot utilissima Pohl. & Mandioca \\
\hline Mansoa standleyi (Steyerm.) A.H.Gentry & Cipó alho \\
\hline Oenocarpus bacaba Mart. & Bacaba \\
\hline Passiflora amalocarpa Barb. Rodr. & Maracujá \\
\hline Peperomia pellucida (L.) Kunth & Lingua de sapo \\
\hline Plinia cauliflora (Mart.) Kausel & Jabuticaba \\
\hline Portulaca pilosa L. & Amor crescido \\
\hline Rollinia mucosa (Jacq.) Baill & Biribá \\
\hline Smilax lutescens Vell. & Pitanga \\
\hline Solanum paniculatum L. & Jurubeba \\
\hline Spondias mombin L. & Cajá \\
\hline Stachytarpheta cayennensis (Rich.) Vahl & Gervão \\
\hline $\begin{array}{c}\text { Syagrus romanzoffiana (Cham.) Glassman } \\
\text { Talisia acutifolia Radlk. }\end{array}$ & $\begin{array}{l}\text { Coquinho } \\
\text { Pitomba }\end{array}$ \\
\hline Theobroma grandiflorum (Willd. ex. Spreng) & \\
\hline K.Schum. & Cupuaçu \\
\hline $\begin{array}{l}\text { Trifolium polymorphum Poir. } \\
\text { Xylopia aromatica (Lam.) Mart. }\end{array}$ & $\begin{array}{c}\text { Trevo } \\
\text { Pimenta de macaco }\end{array}$ \\
\hline
\end{tabular}


Abelmoschus esculentus (L.) Moench Acmella oleracea (L.) R.K. Jansen Allium schoenoprasum $\mathrm{L}$. Aloe vera $\mathrm{L}$.

Amaranthus viridis $\mathrm{L}$. Annona muricata $L$. Annona squamosa $\mathrm{L}$. Arrabidaea chica (Bonpl.) Verl. Artemisia vulgaris $L$. Artocarpus heterophyllus Lam. Averrhoa bilimbi L. Averrhoa carambola $L$. Azadirachta indica A. Juss. Brassica oleracea $L$. Cajanus cajan (L.) Huth Capsicum chinense Jacq. Capsicum frutescens $\mathrm{L}$. Capsicum sp. Carica papaya L. Chenopodium ambrosioides $L$. Chrysobalanus icaco $\mathrm{L}$. Cinnamomum zeylanicum Blume Citrullus lanatus Matsum. \& Nakai. Citrus aurantifolia Swingle Citrus aurantium L.

Citrus limon (L.) Osbeck

Citrus reticulata Blanco Citrus sinensis (L.) Osbeck Cocos nucifera $L$. Coriandrum sativum $\mathrm{L}$. Corylus avellana L. Crescentia cujete $L$. Cucumis melo L. Cucumis sativus $\mathrm{L}$. Cucurbita moschata Duchesne Cucurbita pepo L. Curcuma longa $L$. Cymbopogon citratus Stapf Dioscorea alata $L$.

Dypsis lutescens (H. Wendl.) Beentje \& J. Dransf.

Eruca sativa Mill.

Euphorbia tirucalli L.

Ficus carica L.

Gossypium hirsutum L.

Hibiscus sabdariffa L.

\author{
Quiabo \\ Jambú \\ Cebolinha \\ Babosa \\ Carirú \\ Graviola \\ Ata \\ Pariri \\ Cibalena \\ Jaca \\ Limãozinho \\ Carambola \\ Nim (Mosqueiro) \\ Couve \\ Feijão Guandú \\ Pimenta amarela \\ Pimenta malagueta \\ Pmenta de cheiro \\ Mamão \\ Mastruz \\ Ajuru \\ Canela \\ Melancia \\ Limão galego \\ Laranja da terra \\ Limão \\ Tangerina \\ Laranja \\ Coco \\ Coentro \\ Avelã \\ Cujúba \\ Melão \\ Pepino \\ Abóbora \\ Abobrinha \\ Açafrão \\ Capim santo \\ Cará \\ Palmeira Areca \\ Rúcula \\ Cachorro pelado \\ Fígo \\ Algodão \\ Vinagreira
}


Inga Edulis L.

Jatropha multifida L.

Juniperus chinensis $L$.

Kalanchoe pinnata (Lam.) Pers.

Khaya ivorensis A. Chev.

Lactuca sativa $L$.

Leonotis nepetifolia (L.) R.Br.

Leonurus sibiricus $L$.

Linum usitatissimum L.

Malphigia glabra L.

Mangifera indica $L$.

Melissa officinalis L.

Mentha arvensis $L$.

Mentha spicata $L$.

Morinda citrifolia L.

Morus nigra L.

Musa paradisiaca L.

Ocimum basilicum L.

Ocimum basilicum L.

Ocimum gratissimum L.

Pachycereus pringlei (S. Watson) Britton \& Rose

Pedilanthus tithymaloides (L.) Poit.

Persea americana Mill.

Petiveria alliacea $L$.

Phaseolus vulgaris $\mathrm{L}$.

Piper nigrum L.

Plantago major $\mathrm{L}$.

Plectranthus amboinicus Lour.

Plectranthus barbatus Andrews

Prunus apetala (Siebold \& Zucc.) Franch. \&

Sav.

Prunus cerasus L.

Psidium guajava $\mathrm{L}$.

Punica granatum $\mathrm{L}$.

Ricinus communis $L$.

Rosmarinus officinalis $L$.

Ruta graveolens $\mathrm{L}$.

Saccharum officinarum L.

Sambucus nigra $L$.

Solanum aethiopicum L.

Solanum lycopersicum L.

Spondias dulcis Parkinson

Styrax officinalis $L$.

Symphytum officinale $\mathrm{L}$.

Syzygium cumini (L.) Skeels
Ingá

Mertiolate

Pinheiro (Kaizuza)

Folha santa

Mogno africano

Alface

Cordão de frade

Macaé

Linhaça

Acerola

Manga

Erva cidreira

Vick

Hortelã

Noni

Amora

Banana

Favacão

Manjericão

Favaca

Cacto

Coramina

Abacate

Tipi (Guiné)

Feijão

Pimenta do reina

Transage

Malvarisca

Boldo

Cereja

Ginja

Goiaba

Romã

Mamona

Alecrim

Arruda

Cana de açucar

Sabugueira

Jiló

Tomate

Caja manga

Esturaque

Confrei

Ameixa (Jamelão) 
Syzygium malaccense (L.) Merr. \& L.M. Perry

Tagetes patulaL.

Talinum patens (L.) Willd.

Tamarindus indica $L$.

Theobroma cacao $\mathrm{L}$.

Turnera guianensis $\mathrm{L}$.

Veronica officinalis $L$.

Vigna unguiculata (L.) Walp.

Vitis vinifera $L$.

Zea mays L.

Zingiber officinale Roscoe
Jambo

Crava defunto

João gomes

Tamarindo

Cacau

Chanana

Verônica

Feijão de corda

Uva

Milho

Gengibre

\section{QUESTIONÁRIO P/ QUINTAIS AGROFLORESTAIS \\ (CARACTÉRIZAÇÃO: PLANTAS E QUINTAIS)}

$\mathrm{CAP}=$

Tamanho do quintal=

\section{Nome popular?}

$\mathrm{R}$ :

Procedência da muda:

Doação ( )

Compra ( )

Produzida pelo cultivador ( )

Forma de uso:

Uso religioso ( )

Alimentação ( )

Medicinal ( )

Sombra ( )

Venda ( )

Outro:

\section{Quanto ao manejo:}

Bom - adubação, irrigação e poda ( )

Regular - um ou dois tipos de trato ( )

Ruim - sem nenhum tipo de trato ( ) 


\section{PÓS-ENTREVISTA}

DAP $($ convertido $)=$

Nome científico:

$\mathrm{R}$ :

Hábito de crescimento:

Árvore (

Herbáceo ( )

Arbusto ( )

Arvoreto ( )

Trepadeira ( )

Liana ( )

Subarbustivo ( ) 